\title{
Pattern of Innervation and Recruitment of Different Classes of Motoneurons in Adult Zebrafish
}

\author{
Konstantinos Ampatzis, Jianren Song, Jessica Ausborn, and Abdeljabbar El Manira \\ Department of Neuroscience, Karolinska Institute, 17177 Stockholm, Sweden
}

In vertebrates, spinal circuits drive rhythmic firing in motoneurons in the appropriate sequence to produce locomotor movements. These circuits become active early during development and mature gradually to acquire the flexibility necessary to accommodate the increased behavioral repertoire of adult animals. The focus here is to elucidate how different pools of motoneurons are organized and recruited and how membrane properties contribute to their mode of operation. For this purpose, we have used the in vitro preparation of adult zebrafish. We show that different motoneuron pools are organized in a somatotopic fashion in the motor column related to the type of muscle fibers (slow, intermediate, fast) they innervate. During swimming, the different motoneuron pools are recruited in a stepwise manner from slow, to intermediate, to fast to cover the full range of locomotor frequencies seen in intact animals. The spike threshold, filtering properties, and firing patterns of the different motoneuron pools are graded in a manner that relates to their order of recruitment. Our results thus show that motoneurons in adult zebrafish are organized into distinct modules, each with defined locations, properties, and recruitment patterns tuned to precisely match the muscle properties and hence produce swimming of different speeds and modalities.

\section{Introduction}

In vertebrates, spinal circuits drive rhythmic firing in motoneurons (MNs), leading to muscle contractions that underlie locomotion (Grillner and Jessell, 2009). The propulsive movements are the result of a continuous interaction among locomotor networks, sensory information, and motor apparatus biomechanical properties (Rossignol et al., 2006; Büschges et al., 2011). Although the ability to move manifests early during development, progressive maturation enables adult animals to acquire the flexibility necessary to accommodate an increased behavioral repertoire (Sillar, 2010; Vinay et al., 2010; Kiehn, 2011). However, the mechanisms underlying the reconfiguration of locomotor circuits remain unclear, primarily because such circuits in adult vertebrates are so difficult to access experimentally.

Zebrafish are available at different developmental stages, enabling the study of locomotor circuits and examine how such circuits are refined to accommodate changes in the swimming pattern and muscle composition. At early developmental stages, the myotome contains almost exclusively fast fibers (Waterman, 1969; van Raamsdonk et al., 1982; Devoto et al., 1996; Stellabotte and Devoto, 2007) and the swimming consists of a fast beat-andglide pattern (Drapeau et al., 2002; Lewis and Eisen, 2003). In the adult, the swimming switches to a slow continuous pattern and

Received Feb. 28, 2013; revised May 8, 2013; accepted May 19, 2013.

Author contributions: K.A. and A.E.M. designed research; K.A., J.S., and J.A. performed research; K.A., J.S., J.A., and A.E.M. analyzed data; K.A., J.S., J.A., and A.E.M. wrote the paper.

This work was supported by a grant from the Swedish Research Council and Karolinska Institute. We thank R. Björnfors and S. Grillner for comments and critical discussion of this manuscript.

Correspondence should be addressed to Abdel El Manira, Department of Neuroscience, Karolinska Institute, 171 77 Stockholm, Sweden. E-mail: Abdel.EIManira@ki.se.

DOI:10.1523/JNEUROSCI.0896-13.2013

Copyright $\odot 2013$ the authors $\quad 0270-6474 / 13 / 3310875-12 \$ 15.00 / 0$ slow and intermediate muscle fibers become differentiated (van Raamsdonk et al., 1982; Devoto et al., 1996). Thus, as zebrafish develop, there is a shift from a larval motor pattern relying on fast fibers into an adult pattern involving slow, intermediate, and fast fibers. In larvae, all MNs participate during swimming and are gradually recruited with increased frequency (Fetcho and McLean, 2010). In the adult, we have previously reported that only ventral MNs are recruited at swimming frequencies $\leq 12 \mathrm{~Hz}$ (Gabriel et al., 2011). However, intact zebrafish also produce short periods of high-frequency swimming up to $\sim 21 \mathrm{~Hz}$ (Kyriakatos et al., 2011). Therefore, it remains unclear whether all MNs participate during swimming in adult zebrafish. In addition, it is unknown how the pattern of recruitment of the different MNs relates to the contractile properties of the muscle they innervate and whether there is a differential recruitment of ventral and dorsal MNs at different swimming frequencies.

In this study, we examine how the organization and recruitment pattern of different MNs are related to the properties of their muscle fibers. We first show that MNs are divided into four somatotopically organized motor pools related to the type of muscle they innervate. We then show that only the late-developing secondary $\mathrm{MNs}$ are used during swimming in adult zebrafish, while the first-developed primary MNs contribute only to escape. The secondary MNs consist of three separate modules with firing properties and morphological features organized in a manner that relates to the innervated muscle type. The MNs are deployed in a stepwise manner as a function of increased swimming speed, according to the contractile properties of their muscle. Our results thus show that MNs in adult zebrafish are organized into distinct modules, each with defined locations, properties, and recruitment patterns, tuned to match the muscle properties and hence produce swimming at different speeds and modalities. 


\section{Materials and Methods}

Animals. Juvenile/adult (6-8 weeks postfertilization) wild-type ( $\mathrm{AB} /$ Tübingen and Tupfel long fin-TL) zebrafish (Danio rerio) of either sex were used for this study. All experimental protocols were approved by the local Animal Research Ethical Committee, Stockholm.

Retrograde labeling of MNs. Retrograde labeling of MNs was performed using the fluorescent tracers rhodamine-dextran (molecular weight, 3000; Invitrogen) and Alexa Fluor 488dextran (molecular weight, 10,000; Invitrogen). Crystals of the dyes were first dissolved in distilled water and subsequently desiccated on the tip of sharp tungsten pins, which were then used to inject the tracers into specific muscles. The animals were anesthetized in $0.03 \%$ tricaine methane sulfonate (MS-222, SigmaAldrich). Retrograde tracers were then injected into different muscle fibers with dye-soaked pins to sever the motor axons innervating these muscles and to allow uptake of the dyes. After recovery from anesthesia, the injected animals were kept overnight to allow for retrograde transport of the tracer. To reveal the position of MNs within the motor column, fish were deeply anesthetized with $0.1 \%$ MS-222 solution and intracardially perfused with $4 \%$ paraformaldehyde (PFA) in phosphate buffer (PB, $0.1 \mathrm{M}, \mathrm{pH} 7.4$ ). The spinal cord was then dissected out from the vertebral column and postfixed in $4 \%$ PFA solution overnight at $4^{\circ} \mathrm{C}$ and subsequently rinsed in PBS and coverslipped with antifade fluorescent mounting medium (Vectashield, Vector Labs).

In vitro preparation. The procedure of dissection has been described previously (Gabriel et al., 2008, 2009, 2011; Kyriakatos et al., 2011; Ausborn et al., 2012). Zebrafish were cold anesthetized in a slush of frozen extracellular solution containing MS-222 and eviscerated. The extracellular solution contained (in $\mathrm{mm}$ ) the following: $134 \mathrm{NaCl}, 2.9 \mathrm{KCl}, 2.1$ $\mathrm{CaCl}_{2}, 1.2 \mathrm{MgCl}_{2}, 10$ HEPES, and 10 glucose, $\mathrm{pH} 7.8$, adjusted with $\mathrm{NaOH}$, and an osmolarity of $290 \mathrm{mOsm}$. The brain was then exposed and cut at the level of the midbrain. The epaxial musculature was carefully removed up to the caudal end of the dorsal fin, leaving the musculature of the tail region intact. The skin was gently removed from the tail to reveal the myotomes. The neuronal spines forming the neural arch were removed along with the neural zygapophyses four or five segments rostral to the dorsal fin to expose the spinal cord and allow access with patchclamp recording electrodes. In addition, the Weberian apparatus was removed and the first two segments of the spinal cord were exposed to allow extracellular stimulation of the descending axons. The brainstemspinal cord preparation was placed dorsal side up and fixed with Vaseline to allow for bilateral extracellular recording from the motor nerves. Motor nerve activity was recorded on opposite sides of the animal while different regions in the rostral spinal cord and brainstem were stimulated to induce locomotion. For whole-cell intracellular recordings, the preparation was transferred to a recording chamber, placed lateral side up, and fixed with Vaseline. The chamber was continuously perfused with extracellular saline solution containing tubocurarine (10 $\mu \mathrm{M}$; SigmaAldrich) to block neuromuscular junctions and to eliminate muscle contractions for the whole duration of the experiment. All experiments were performed at an ambient temperature of $20-22^{\circ} \mathrm{C}$.

Electrophysiology. Extracellular recording and stimulation electrodes were pulled from borosilicate glass (outer diameter, $1 \mathrm{~mm}$; inner diameter, $0.87 \mathrm{~mm}$; Harvard Apparatus) on a Flaming/Brown microelectrode puller (P-87, Sutter Instruments), broken down to the desired tip diameter $(15-25 \mu \mathrm{m})$, and fire polished. Extracellular recordings were performed from the motor nerves running through the intermyotomal clefts at the tail, where the musculature was left intact. A stimulating electrode was placed either on the dorsal part of the rostral spinal cord or at the Mauthner cell region in the brainstem to elicit slow or escape/fast locomotor episodes, respectively. Extracellular signals were amplified (gain, 10,000 ) with a differential AC amplifier (AM Systems) and filtered with low and high cutoff frequencies of $300 \mathrm{~Hz}$ and $1 \mathrm{kHz}$, respectively. Intracellular whole-cell recordings were performed from identified MNs prelabeled with a retrograde tracer. For intracellular recordings, electrodes (resistance, 9-13 M 2 ) were pulled from borosilicate glass (outer diameter, $1.5 \mathrm{~mm}$; inner diameter, $0.87 \mathrm{~mm}$; Hilgenberg) on a vertical puller (PC-10 model, Narishige) and filled with intracellular solution containing (in $\mathrm{mM}$ ) the following: $120 \mathrm{~K}$-gluconate, $5 \mathrm{KCl}, 10$ HEPES, 4 $\mathrm{Mg}_{2}$ ATP, $0.3 \mathrm{Na}_{4} \mathrm{GTP}, 10 \mathrm{Na}$-phosphocreatine, $\mathrm{pH}$ 7.4, adjusted with $\mathrm{KOH}$, and osmolarity of $275 \mathrm{mOsm}$. Dextran-labeled MNs were visualized using a fluorescence microscope (Axioskop FS Plus, Zeiss) equipped with IR-differential interference contrast optics and a CCD camera with frame grabber (Hamamatsu) and were then targeted specifically. Intracellular patch-clamp electrodes were advanced into the exposed portion of the spinal cord through the meninges using a motorized micromanipulator (Luigs \& Neumann) while applying constant positive pressure. Intracellular signals were amplified with a MultiClamp 700B intracellular amplifier (Molecular Devices) and low-pass filtered at $10 \mathrm{kHz}$. In current-clamp recordings, no bias current was injected. Only MNs that had stable membrane potentials $\leq-48 \mathrm{mV}$, fired action potentials to suprathreshold depolarizations, and showed minimal changes in series resistance $(<5 \%)$ were included in this study. Finally, for labeling of the recorded $\mathrm{MNs}, 0.5 \%$ neurobiotin tracer (Vector Labs) was added to the intracellular solution.

Neurobiotin histochemistry. During intracellular whole-cell recordings ( $\sim 30 \mathrm{~min})$, neurons were passively filled with neurobiotin tracer to reveal their morphological characteristics post hoc. Spinal cords with neurobiotin-filled MNs were dissected out of the vertebral column and transferred into $4 \%$ PFA solution overnight at $4^{\circ} \mathrm{C}$. The tissue was then washed extensively with PBS and incubated in streptavidin conjugated to Alexa Fluor 488 (1:500; Invitrogen) in PBS containing 0.5\% Triton X-100 (Sigma-Aldrich) and $2 \%$ normal goat serum overnight at $4^{\circ} \mathrm{C}$. Subsequently, the tissue was washed five times (5 min each) in PBS and 

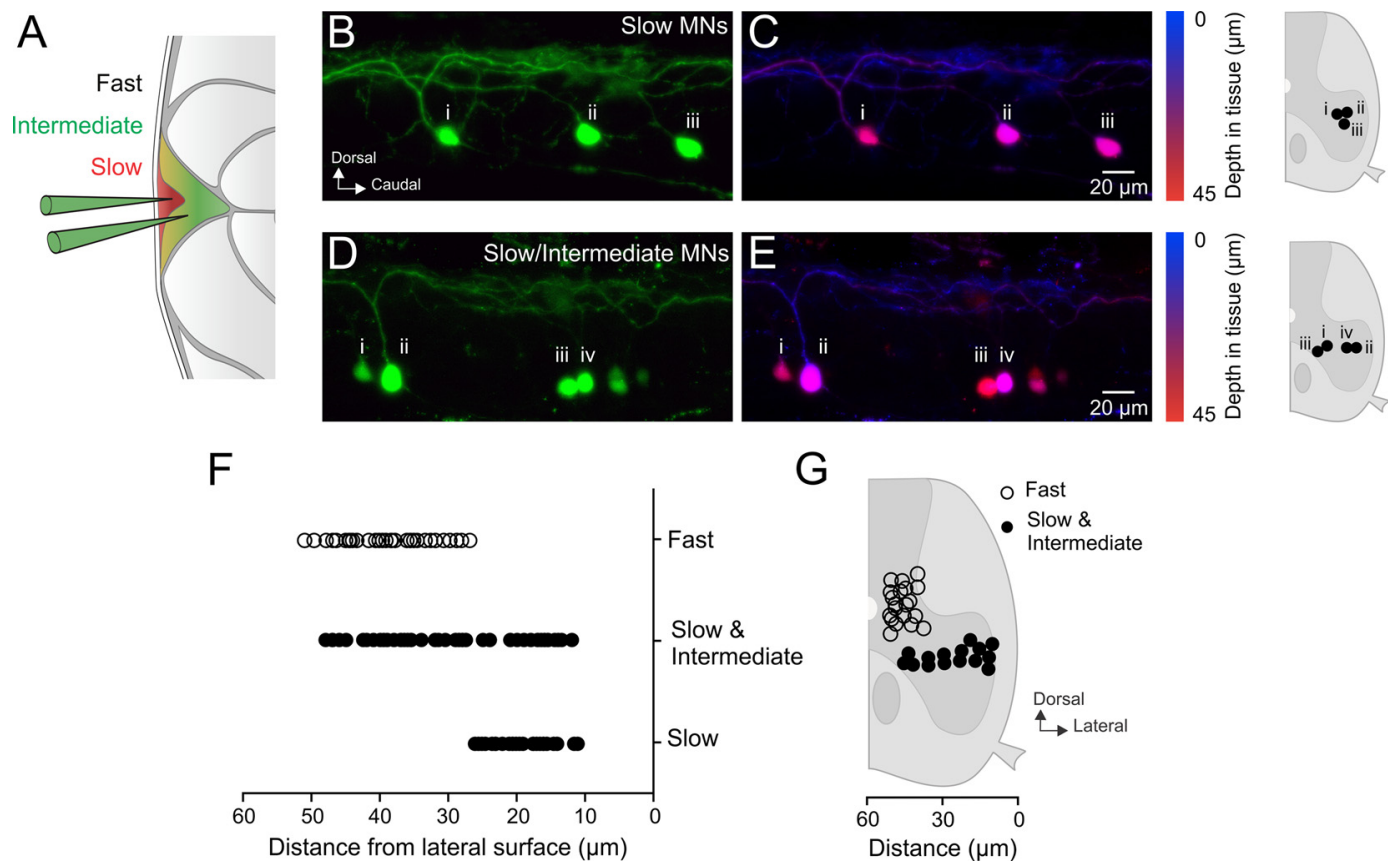

Figure 2. Somatotopic organization of MNs in adult zebrafish. $A$, Schematic showing the sites of injections into the different parts of the axial muscles (red, slow; green, intermediate; gray, fast). $B$, Slow MNs labeled by injection of slow muscle. C, Depth analysis shows that slow MNs are located in the lateral aspect of the motor column. $\boldsymbol{D}$, Slow and intermediate MNs labeled by injection of a fluorescent dye in slow and intermediate muscles. $\boldsymbol{E}$, The labeled MNs are located in the ventral aspect of the motor column and extend along the lateromedial axis. $\boldsymbol{F}$, Graph showing the distance of the MNs retrogradely labeled by injecting a fluorescent dye in slow, intermediate/slow, and fast muscle. G, Position of the different MNs in the motor column labeled by injections into only slow muscles, into slow and intermediate muscles, and into fast muscles. The dorsoventral position of the different MNs is set relative to the Mauthner axon and dorsal edge of the spinal cord.

mounted on glass slides and coverslipped with fluorescent mounting medium.

Data analysis. For all the electrophysiological recordings, data analysis was performed using Spike2 software (Cambridge Electronic Design) or Clampfit (Molecular Devices). The electrophysiological data were analyzed to calculate several parameters, such as the input resistance, the firing threshold, the spike peak amplitude, the spike width, the afterhyperpolarization or afterdepolarization amplitude, the minimum recruitment frequency, and the instantaneous firing frequency. In particular the input resistance of MNs was calculated as the slope of a regression line to the linear region of the $I-V$ curve (membrane potentials of -80 to -60 $\mathrm{mV}$ ), which was obtained by series injection of hyperpolarizing current pulses (duration, $500 \mathrm{~ms}$ ). The action potential voltage threshold of each $\mathrm{MN}$ was determined from the measured membrane potential at which the $d V / d t$ exceeded $10 \mathrm{Vs}^{-1}$. The minimum recruitment frequency of MNs was defined as the slowest burst frequency of a swimming episode at which the neurons discharged action potentials. Moreover, the minimum locomotor frequency obtained during swimming episodes varied between preparations. In most of the fast-swimming stimulations, the lowest frequency obtained was not the minimum frequency at which the cells are recruited. Finally, the instantaneous firing frequency was obtained for each $\mathrm{MN}$ by calculating the action potential events as a fraction of time.

For the whole-mount imaging of the spinal cords, a laser scanning confocal microscope (LSM 510 Meta, Zeiss) was used. The position of retrogradely labeled MNs was examined in completely isolated whole spinal cords mounted on the lateral side. To control that the spinal cords were correctly positioned on the lateral side, we ensured the parallel alignment of the two Mauthner axons and their relative position to the central canal. Motoneuron distribution was analyzed in 2-3 segments rostral to the dorsal fin that correspond to the region where intracellular recordings were made. The lateral edge of the spinal cord was set as the zero point of the lateromedial axis. The dorsoventral position was set relative to the Mauthner axon and the dorsal edge of the spinal cord. Neurobiotin-filled MNs were photographed and the soma area of neurons was measured based on these microphotographic images using ImageJ software (http://imagej.nih.gov/ij/). The neuronal processes (axons and dendrites) of each $\mathrm{MN}$ were then traced using a semiautomated tracing software, NeuronJ (Meijering et al., 2004; ImageJ plugin software). To describe in detail the MN dendritic arborization, Sholl analysis (Sholl, 1953) was performed on the reconstructed neurons using the Sholl Analysis Plugin for ImageJ (http://rsbweb.nih.gov/ij/). Finally, we used a previously described method (Bender et al., 2003) to obtain twodimensional density maps of the dendrites from each $\mathrm{MN}$ pool. Reconstructed dendritic processes from each cell of the same group were aligned by soma position (epicentrum) and digitally superimposed. The dendritic arbors were expressed as line density based on these image reconstructions. The images were subsequently smoothed using a Gaussian blur filter in Photoshop CS6 (Adobe Systems) to show the mean shape of projections. Then, color-coded contour maps were constructed using OriginPro (OriginLab). Principal component analysis (PCA) was applied to the data using XLStat software (Addinsoft). Additional statistical analysis was performed using a one-way ANOVA (Prism 4.0, GraphPad Software) followed by Tukey's post hoc tests. Differences were considered to be significant if $p<0.05$. All data presented here are given as mean $\pm \mathrm{SEM}$.

\section{Results}

\section{Somatotopic organization of distinct MN pools}

In adult zebrafish, slow and fast muscle fibers occupy distinct regions of the axial muscle (van Raamsdonk et al., 1982; Devoto et al., 1996). Fast muscle fibers represent the deep and large portion of the ventral and dorsal myotomes, while slow muscle fibers are segregated into a lateral wedge-shaped region of the myotomes at the level of the horizontal septum, which separates the ventral (hypaxial) and dorsal (epaxial) muscles (Figs. 1, 2A). In addition, there are intermediate muscle fibers located between the slow and fast muscle fibers. To determine whether the MNs innervating the different compartments of the myotomes are somatotopically organized, we performed a series of retrograde labeling experiments (Fig. 1). MNs innervating fast muscles were labeled by injections of fluorescent dyes into the deep hypaxial 
and epaxial muscles, while those innervating slow and intermediate muscles were labeled by injections into the lateral slow and intermediate muscles (Fig. 1). Selective injections ( $n=12$; Fig. $1 A)$ of different fluorescent dyes into the epaxial (green) and hypaxial (red) fast muscles labeled primary MNs (pMNs) and a proportion of secondary MNs (sMNs) located in the dorsal part of the motor column (Fig. 1B). Our injections labeled the middle pMN (Fig. $1 B$, MiP, red) innervating the epaxial fast muscle and the caudal pMN (Fig. 1B, CaP, green) innervating the ventral part of the hypaxial fast muscle (Fig. 1B). In contrast, the dorsally located sMNs did not show any obvious rostrocaudal segregation in the spinal cord segment related to the part of the myotome they innervate (Fig. $1 A, B$ ).

Injections of rhodamine dextran into slow and intermediate muscles $(n=15)$ retrogradely labeled only sMNs that form a narrow strip located in the most ventral area of the motor column (Fig. 1C,D). To confirm the relative location of MNs innervating slow and intermediate muscles in relation to those innervating fast ones, different dyes were injected selectively into fast (green) and slow/intermediate (red) muscles $(n=8)$. No double-labeled MNs were observed (Fig. 1E,F), indicating that the injections were restricted to the target muscles. In all experiments, MNs innervating fast muscles were always located more dorsally than those innervating slow/intermediate muscles (Fig. $1 F$ ). These results show a dorsoventral organization of the $\mathrm{MN}$ pools related to the contractile properties of the muscles they innervate.

The ventral aspect of the motor column in adult zebrafish extends laterally. To reveal the position of the slow and intermediate MNs along the mediolateral axis, a fluorescent dye was either injected exclusively into the superficial slow muscle or into the deep aspect of the myotomes at the level of the horizontal septum to target both slow and intermediate muscle fibers (Fig. $2 A$ ). The labeled MNs were reconstructed using confocal microscopy and their mediolateral position was determined. Fluorescent dye injections into slow muscles labeled only MNs in the ventrolateral part of the motor column (Fig. $2 B, C$ ). Intermediate sMNs were labeled by dye injections into the deeper layers of the myotomes, where intermediate muscle fibers are located (Fig. $2 A$ ). Such injections labeled both slow and intermediate MNs that were located in the ventral aspect of the motor column and extended along the lateromedial axis (Fig. $2 D, E$ ). The analysis of the lateromedial position of the different retrogradely labeled MNs showed that injections into the slow muscle compartment only labeled MNs located in the lateral part of the motor column. Injections into the deep parts of the lateral muscle labeled both lateral (slow) and medial (intermediate) MNs (Fig. $2 F, G$ ). In contrast, injections into fast muscles only labeled dorsal pMNs and sMNs located in the dorsomedial part of the motor column (Fig. $2 F, G$ ). These results show that there is a somatotopic organi- zation of the MNs into distinct pools depending on the type of muscles they innervate.

\section{Frequency-dependent recruitment of slow and fast MNs}

To determine whether all MN pools are recruited during swimming and whether the slow-intermediate pools become derecruited at fast swimming speeds, we recorded from MNs from each pool while swimming activity was induced by electrical stimulation (Fig. 3A). For this, we used the in vitro brainstem/ spinal cord preparation of juvenile/adult zebrafish in which the different types of MNs were prelabeled by injecting a fluorescent dye into the different muscle compartments (Fig. $3 A, B$ ). In freely swimming adult zebrafish, the frequency of locomotion ranges from $\sim 1$ to $21 \mathrm{~Hz}$ with the peak of activity $\sim 8-10 \mathrm{~Hz}$ (Kyriakatos et al., 2011). The in vitro brainstem/spinal cord preparation did not display spontaneous swimming/escape activities. Therefore, different areas in the rostral spinal cord and brainstem were stimulated to produce swimming at frequencies corresponding to those seen in vivo (Fig. $3 A$ ). Long-lasting episodes of continuous swimming with frequencies $\leq 12 \mathrm{~Hz}$ were induced by stimulation at the junction between the brainstem and spinal cord (Fig. 3A,C, stimulation site 2). At these frequencies, only ventral MNs (slow and intermediate) were recruited while dorsal MNs (fast) only received subthreshold locomotor-related membrane potential oscillations (data not shown; Gabriel et al., 2011). To determine whether the dorsal MNs would be recruited at swimming frequencies $>12 \mathrm{~Hz}$, we stimulated 

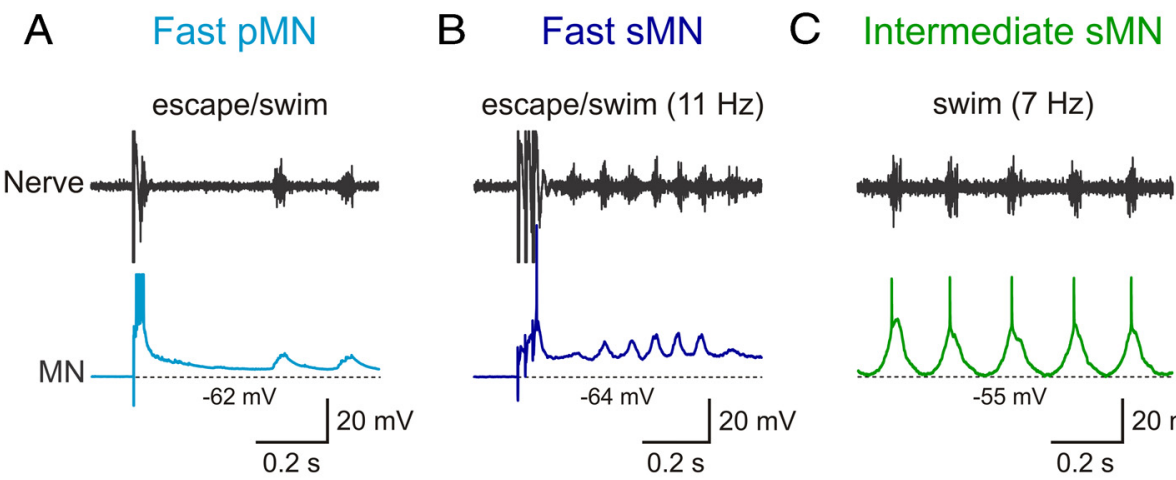

D Slow sMN
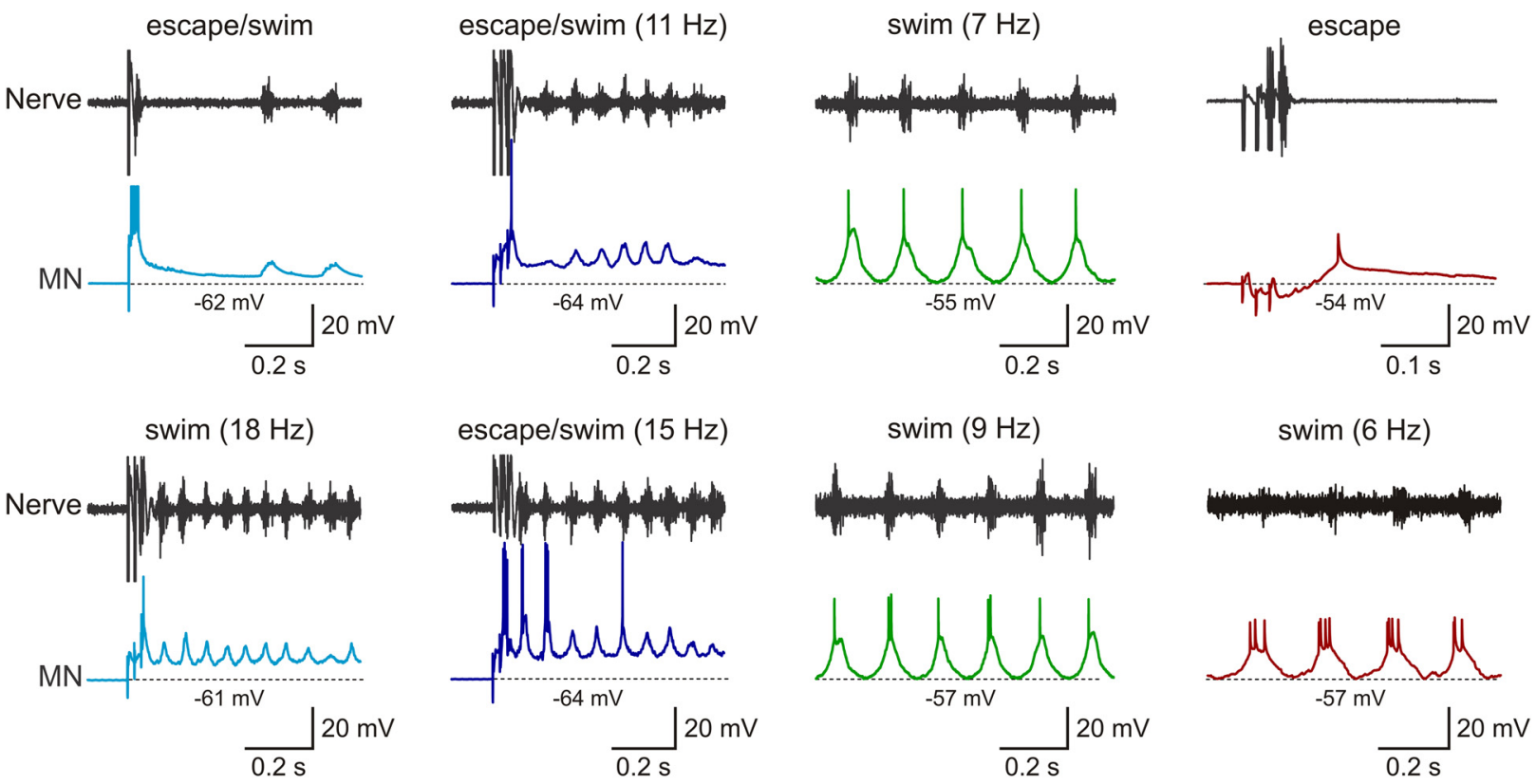

$\operatorname{swim}(9 \mathrm{~Hz})$

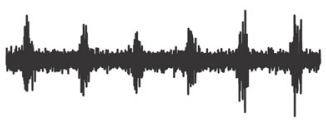

swim $(6 \mathrm{~Hz})$
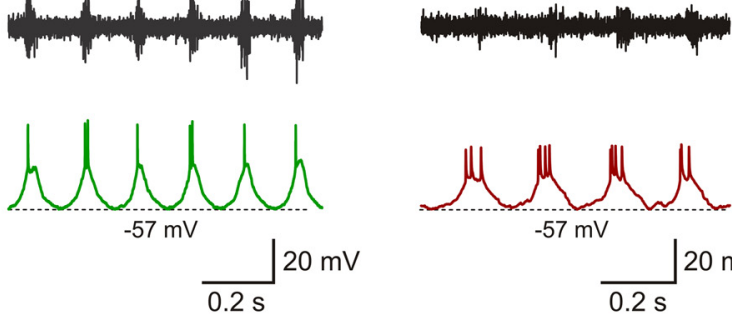

$\operatorname{swim}(25 \mathrm{~Hz})$
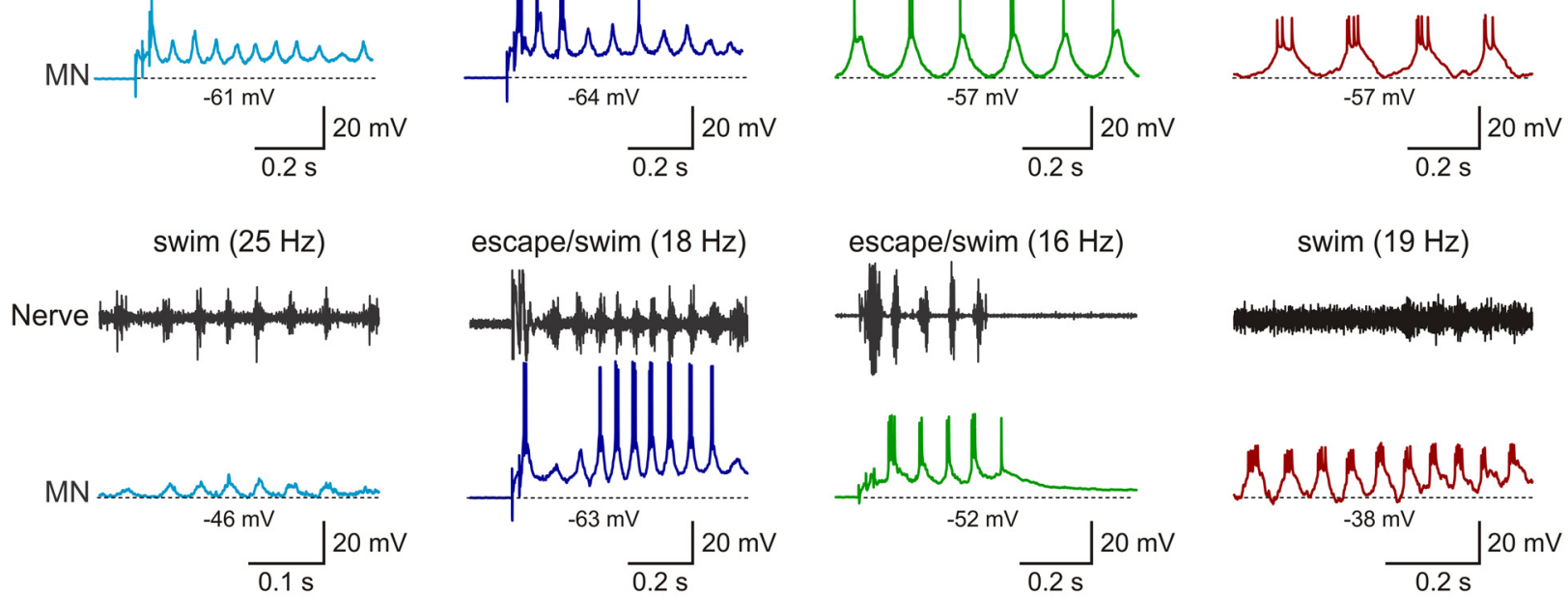

$\operatorname{swim}(19 \mathrm{~Hz})$
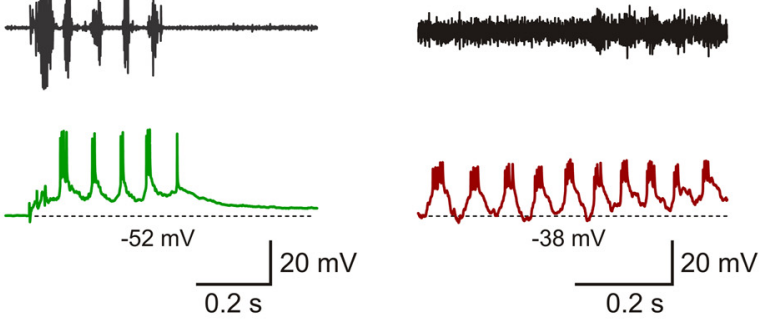

E

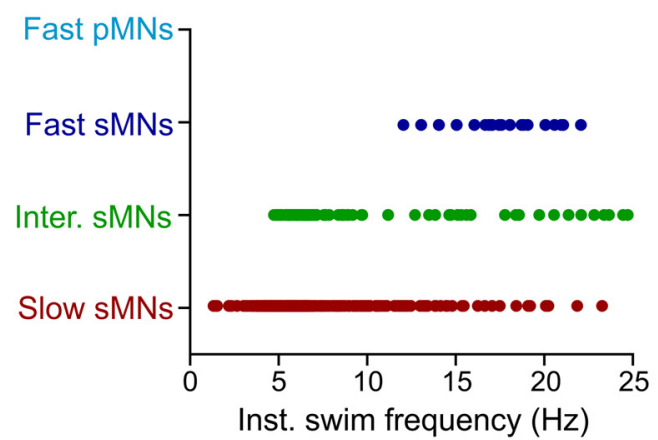

Figure 4. Frequency-dependent recruitment pattern of the different types of MNs. $A$, Fast pMNs are only recruited during escape induced by stimulation at the Mauthner cell region, but are never active during swimming, even at very high frequencies. $\boldsymbol{B}$, Fast sMNs are recruited during the escape burst and also during swimming at high frequencies. $\boldsymbol{C}$, Intermediate $M N s$ are recruited both at slow and fast swimming frequencies. $\boldsymbol{D}$, Slow MNs are not active during escape. They are recruited during slow swimming and do not become derecruited at high swimming frequencies. $\boldsymbol{E}$, Graph showing recruitment of the different MNs as a function of the instantaneous swimming burst frequency. Secondary MN pools are recruited in a stepwise manner. Individual data points represent the instantaneous swimming frequencies of all swimming cycles where the respective MN produced at least one action potential.

different brainstem areas (Fig. 3A). We found that stimulation in the region close to the Mauthner cell (Fig. $3 A$, stimulation site 1 ) induced short bouts of swimming activity at high frequencies $(6-32 \mathrm{~Hz})$ that were preceded with a large motor burst on the contralateral motor nerve, presumably corresponding to escape (Fig. 3D).

To determine the activity pattern of the different MNs during high-frequency swimming and presumed escape, prelabeled MNs were targeted with visually guided patch-clamp electrodes while the locomotor activity was monitored by recording from a motor nerve (Fig. $3 A$ ). The fast pMNs $(n=6)$ always fired action potentials only during the strong "escape" burst occurring after the stimulation, but were never recruited during swimming activity, even at very high frequencies (Fig. 4A). The fast sMNs $(n=6)$ also fired action potentials during the presumed escape burst. These MNs received sub- 
threshold membrane potential oscillations at frequencies $<15 \mathrm{~Hz}$ (Fig. $4 B$ ) and became recruited only when the swimming frequency exceeded $\sim 15 \mathrm{~Hz}$. They displayed more consistent firing during consecutive swimming bursts at higher frequencies (e.g., $18 \mathrm{~Hz}$; Fig. $4 B$, bottom). In contrast, the intermediate $(n=6)$ and slow $(n=9)$ sMNs were recruited at much lower frequencies and were not derecruited at higher swimming frequencies (Fig. 4C,D).

During swimming, only sMNs were recruited (Fig. 4E). At frequencies $<4 \mathrm{~Hz}$, swimming activity was exclusively produced by recruitment of the slow sMNs and the intermediate sMNs were deployed only when the frequency exceeded this level (Fig. 4E). The fast dorsal sMNs became recruited only at frequencies exceeding $\sim 12-15 \mathrm{~Hz}$ (Fig. $4 E$ ). There is thus a stepwise recruitment of $\mathrm{MNs}$ as a function of swimming frequency from those innervating slow, to intermediate, to fast muscles and none of the earlyactivated MNs become derecruited at higher frequencies.

These results show that swimming ac-

tivity is exclusively mediated by sMNs. The different types of sMNs are deployed in a stepwise manner with increased swimming frequency, depending on the properties of the muscle fibers they innervate. In addition, we show that the fast sMNs are shared between escape and swimming circuits, in contrast to slow and intermediate sMNs and fast pMNs, which are exclusively dedicated to swimming or escape, respectively.

\section{Cellular and firing properties of spinal MNs}

The muscle innervation pattern clearly defines distinct pools of MNs innervating fast, intermediate, and slow muscles. This raises the question whether the intrinsic properties of each $\mathrm{MN}$ pool are unique or whether they are organized in a continuum from the very slow to the fast ones. To assess this, we analyzed the action potential waveform and firing properties of the different MN pools in quiescent preparations before eliciting swimming/escape activities. We first examined the action potential waveform by injecting short $(0.5 \mathrm{~ms})$ depolarizing current steps that displayed clear differences among the different MN pools (Fig. $5 A$ ). The action potential threshold was different among the different groups of MNs (Fig. 5B; 1-way ANOVA, $F_{(3,78)}=82.8, p<$ $0.0001)$, being lowest in slow sMNs $(-36.2 \pm 1.2 \mathrm{mV} ; n=24)$ and increasing gradually in intermediate sMNs $(-31.7 \pm 0.4 \mathrm{mV}$; $n=17)$, fast sMNs $(-24.8 \pm 0.5 \mathrm{mV} ; n=27)$ and fast pMNs $(-14.3 \pm 1.6 \mathrm{mV} ; n=14$; Fig. $5 B)$. The action potential amplitude and duration were also significantly different among the different MN types (Fig. 5C,D; 1-way ANOVA, $F_{(3,34)}=180.5$, $p<0.0001)$. In fast $\mathrm{pMNs}$ and fast sMNs, the action potentials were always overshooting with a peak amplitude of $29.9 \pm 1.3$ $\mathrm{mV}$ and $4.6 \pm 1.05 \mathrm{mV}$, respectively (Fig. $5 \mathrm{C}$ ). In addition, these MNs displayed narrow action potentials (half-width fast $\mathrm{pMN}$, $0.4 \pm 0.01 \mathrm{~ms}$; half-width fast sMN, $0.64 \pm 0.03 \mathrm{~ms}$; Fig. $5 D)$ and a large afterhyperpolarization (AHP; fast pMN, $12.3 \pm 0.8 \mathrm{mV}$; fast sMN, $7.4 \pm 0.6 \mathrm{mV}$; Fig. $5 E$ ). In contrast, the action potentials in slow and intermediate sMNs were never overshooting and had a peak amplitude of $-17.7 \pm 0.8 \mathrm{mV}$ and $-10.6 \pm 0.5 \mathrm{mV}$,
B

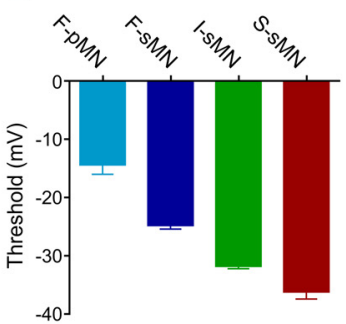

C

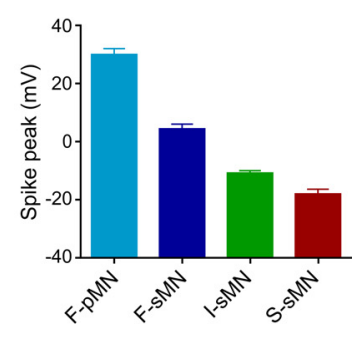

E
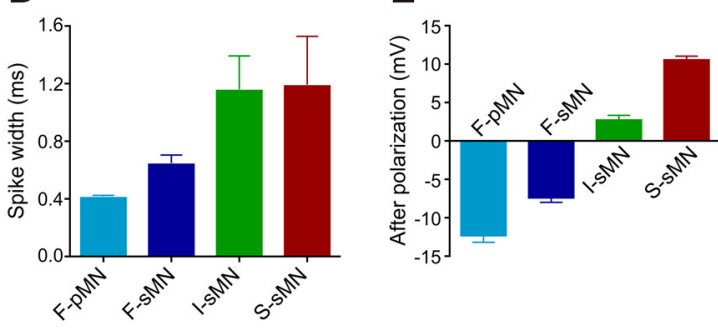

ms

Slow sMN

perties in the different MN pools. $\boldsymbol{A}$, The shape of action potentials differ between the different MN pools. Each trace is an average of 30 sweeps with the stimulation artifact subtracted. Bottom traces show normalized 列 showing the differences in the peak amplitude of the action potentials in the different MN pools. $\boldsymbol{D}$, Graph showing the action ent MNs. $\boldsymbol{E}$, Graph showing the mean afterhyperpolarization amplitude in the different MNs.

respectively. These action potentials were broader (1-way ANOVA, $\left.F_{(3,34)}=3.28, p<0.05\right)$ than those of fast pMNs and fast sMNs (slow sMNs, $1.18 \pm 0.2 \mathrm{~ms}$; intermediate sMNs, $1.15 \pm$ $0.1 \mathrm{~ms}$; Fig. 5D) and were followed by an afterdepolarization (ADP) in slow sMNs (slow sMNs, $10.6 \pm 0.5 \mathrm{mV}$; intermediate sMNs, $2.7 \pm 0.6 \mathrm{mV}$; Fig. 5E).

The difference in the action potential waveform, AHP, and ADP among the different MN pools should affect their repetitive firing differently. To assess whether the repetitive firing differs among the different $\mathrm{MN}$ pools, long depolarizing current steps (1 s) were used (Fig. 6). As predicted by their large AHP, fast pMNs and fast sMNs displayed strong spike frequency adaptation, which terminated their firing prematurely (Fig. 6A,B). The intermediate sMNs fired tonically during depolarizing current pulses and showed less pronounced spike frequency adaptation (Fig. 6C). In contrast, the slow sMNs displayed bursting properties with membrane potential oscillations that increased in frequency as a function of the injected current (Fig. 6D). In addition to a difference in their firing pattern, the different $\mathrm{MN}$ pools also displayed differences in their peak firing frequency (Fig. 6E).

These results show that different types of MNs display distinct spike thresholds, waveforms, and firing patterns that are correlated with the type of muscle fibers they innervate and their order of recruitment with increased swimming speed. Fast MNs display high firing thresholds and a strong spike frequency adaptation, while slow MNs possess low thresholds and a bursting firing pattern.

Frequency-dependent filtering properties of MNs innervating fast and slow muscle fibers

The different MN pools need to operate at different frequency ranges, which could be supported by passive filtering capacities endowed by their intrinsic properties. To assess whether there is a difference in the firing reliability between the different $\mathrm{MN}$ pools, sinusoidal command currents of different frequencies were used (Fig. 7). The firing reliability was differently distributed among 

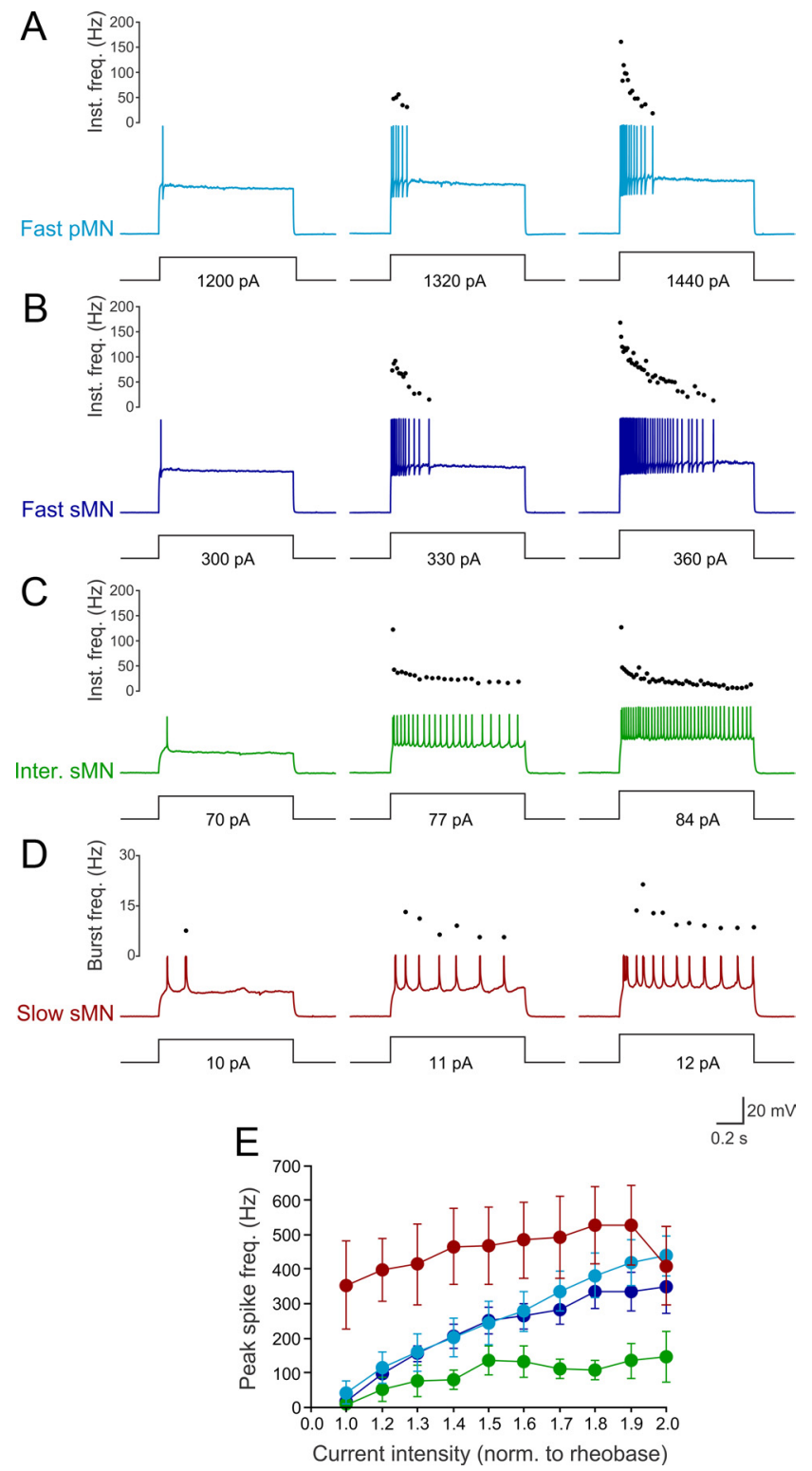

Figure 6. The different MN pools display distinct firing patterns. $\boldsymbol{A}$, Fast pMNs show strong spike frequency adaptation even at high stimulation intensities. $\boldsymbol{B}$, Fast sMNs also display strong spike frequency adaptation that terminates their firing before the end of the stimulation current step. C, Intermediate sMNs fire continuously in response to depolarizing current pulses. $\boldsymbol{D}$, Slow sMNs fire in bursts of action potentials that increase in frequency as a function of the strength of the stimulation current. $\boldsymbol{E}$, Plot showing the frequency between the two first action potentials in the different pools of MNs.

MNs innervating fast, intermediate, and slow muscle fibers. Fast pMNs $(n=5)$ showed low firing reliability to sinusoidal current injections at low frequencies while higher frequencies elicited action potentials more reliably (Fig. $7 A, B$ ). The amplitude of membrane potential deflections in these MNs, however, remained relatively constant at different frequencies, indicating no or weak passive filtering in fast pMNs (Fig. 7C). The fast sMNs $(n=9)$ fired reliably at lower frequencies and their firing decreased with increasing frequency up to $\sim 40 \mathrm{~Hz}$, where they ceased firing (Fig. $7 A, B$ ). The decrease in the firing reliability in these MNs was associated with an increased passive filtering that was more pronounced at sinusoidal current frequencies $>40 \mathrm{~Hz}$ (Fig. $7 C)$. The intermediate $(n=5)$ and the slow $(n=5)$ sMNs fired reliably at low sinusoidal current frequencies, but could not follow frequencies $>15$ and $10 \mathrm{~Hz}$, respectively (Fig. $7 A, B$ ). These sMNs also showed a strong passive filtering with a decrease in the membrane potential oscillation amplitude as a function of the sine wave current frequency (Fig. $7 C$ ). These results show that the reliability of firing and passive filtering properties of the different $\mathrm{MN}$ pools is specifically tuned and is correlated with the contractile properties of the corresponding muscles. The current injected in these experiments was injected in the soma, while the synaptic currents underlying locomotor activity are mostly occurring in the dendrites. This most likely explains why the filtering capacity of slow and intermediate MNs is not manifested during swimming. It seems that the intrinsic filtering at higher frequencies in the sMNs is balanced by an increase in synaptic inputs to maintain their recruitment at swimming frequencies beyond those producing passive filtering.

\section{Morphological features of different MNs}

Finally, we examined whether the different MN pools also exhibit distinct morphological features associated with their location in the motor column, their peripheral pattern of innervation, and their physiological properties. For this, we recorded from the prelabeled fast pMNs and sMNs, intermediate sMNs, and slow sMNs retrogradely labeled by injecting a fluorescent dye into different parts of the myotome (Fig. 1). The MNs were then filled with neurobiotin from the recording electrode and subsequently reconstructed using confocal microscopy. There was a clear difference in the soma size and dendritic morphology among fast pMNs, fast sMNs, intermediate sMNs, and slow sMNs (Fig. 8A; 2 representative examples of each $\mathrm{MN}$ type). The reconstructed MNs were aligned according to their soma position and superimposed two-dimensionally and examined using Sholl analysis (Fig. 8 B,D). Fast pMNs had large somata $\left(167.7 \pm 13.6 \mu \mathrm{m}^{2} ; n=\right.$ $6)$ and displayed considerable dendritic arborizations (1098 \pm $26.2 \mu \mathrm{m}, n=6$ ), which exceeded the length of the segment of origin (Fig. $8 A-D)$. Similarly, fast sMNs $(n=7)$ had relatively large somata $\left(69.1 \pm 4.3 \mu \mathrm{m}^{2} ; n=7\right)$ and extensive dendrites $(324.8 \pm 52.6 \mu \mathrm{m} ; n=7)$ that also exceeded the length of the parent segment to reach one ( $80 \%$ of fast sMNs) or both adjacent segments $(20 \%$ of fast sMNs; Fig. $8 A-D)$. In contrast, ventrally located sMNs had smaller somata (1-way ANOVA, $F_{(3,34)}=$ 56.56, $p<0.0001$; intermediate sMNs, $41.7 \pm 5.3 \mu \mathrm{m}^{2}, n=4$; slow sMNs, $\left.41.2 \pm 3.6 \mu \mathrm{m}^{2}, n=6\right)$, and their dendritic arborizations were less extensive (1-way ANOVA, $F_{(3,34)}=67.67, p<$ 0.0001 ; intermediate sMNs, $149.1 \pm 11.3 \mu \mathrm{m}, n=4$; slow sMNs, $192.4 \pm 14.9 \mu \mathrm{m}, n=6)$. The two types of fast MNs have dorsally arranged dendrites, which extend outside the segment of origin, and a set of ventrally directed dendrites. The innervation field of the latter overlaps with those of the intermediate and slow sMNs that extend dorsally of the cell body. The dendritic arborization of these slow and intermediate sMNs were always confined to the segment of origin (Fig. $8 A-D$ ).

Adult zebrafish MN pools innervating fast, intermediate, and slow muscle fibers each display distinct morphological features. MNs innervating fast muscle fibers are located dorsally and comprise all pMNs and dorsal sMNs. These MNs have extensive dendritic arborizations, which enables them to sample information from neighboring segments. Intermediate and slow muscle fibers are innervated by ventral sMNs located in the ventromedial and ventrolateral aspects of the motor column, respectively. These MNs have dendritic arborizations limited to the parent segment. 
Distinct MN pools with specific

physiological and morphological properties

In adult zebrafish, MNs seem to be segregated into distinct pools with defined somatotopic, physiological, morphological, and recruitment patterns that match the type of muscles they innervate. To confirm that the different MNs can be segregated into distinct pools independent of their somatotopic organization and muscle innervation, PCA was undertaken. The first PCA used morphological features from all the MNs reconstructed after neurobiotin labeling $(n=38)$. The different morphological variables used included the soma size, axonal length, number of the dendritic branch points, dendritic length in both home and in neighboring segments, and the total dendritic length and field. This PCA yielded two principal components, which accounted for $92.45 \%$ of the variance. This analysis revealed a large and heterogeneous cluster of intermingled MNs encompassing all the sMNs and clearly segregated from a pMN cluster (Fig. 9A). Thus, morphological variables allow the distinction between two apparent clusters of MNs, one representing pMNs and the other sMNs. The second PCA ( $n=38 \mathrm{MNs}$ ) used cellular properties that included resting membrane potential, input resistance, rheobase, firing threshold, peak of action potential, half-width duration, the amplitude of the locomotor-related membrane potential oscillations at $4-5 \mathrm{~Hz}$, and minimum recruitment frequency. The first two principal components accounted for $74.80 \%$ of the variance and revealed three or possibly four clusters corresponding to slow/intermediate sMNs, fast sMNs, and fast pMNs (Fig. 9B). The final PCA combined both electrophysiological and morphological properties. The first two principal components accounted for $79.22 \%$ of the variance and revealed four distinct $\mathrm{MN}$ clusters. Thus, the clustering of MNs into four functionally distinct pools requires that both morphological and physiological features are considered and none of these properties alone was able to segregate the MNs into four pools.

\section{Discussion}

We show here that adult zebrafish MNs innervating slow, intermediate, and fast muscles are organized somatotopically in different parts of the spinal motor column. The topographic segregation of these MNs is also mirrored by their biophysical, morphological, and physiological features. Swimming activity is mediated by the recruitment of the late-developed sMNs, while the early-developed pMNs do not participate in swimming and are only active during escape (Fig. 9D). Each MN type forms a discrete module with a precise frequency threshold for recruitment. During swimming, the different types of sMNs are recruited in a stepwise manner at specific frequency thresholds, and the MN pools recruited early remain active as the faster pools are added with increasing swimming speed.
Fast sMN

Intermediate sMN

Slow sMN
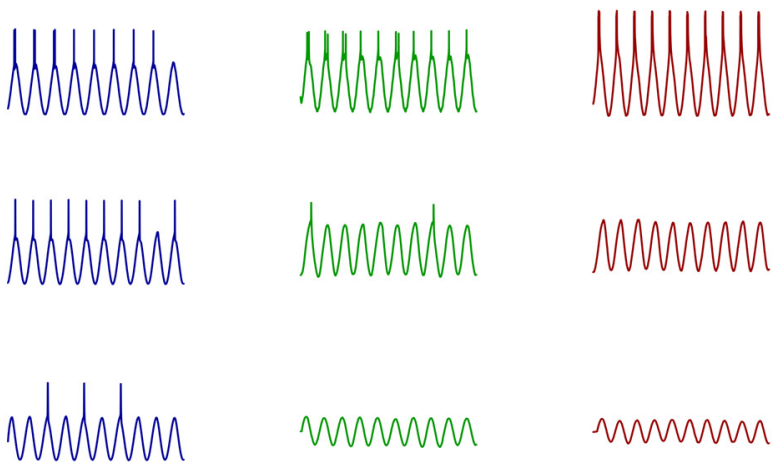

WWWM

MWWM

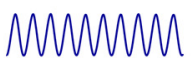

I $20 \mathrm{mV}$
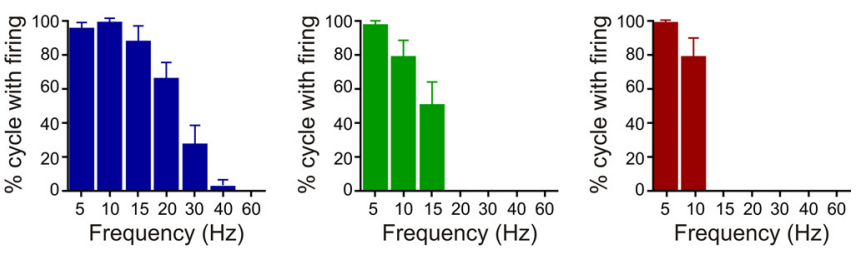

WWWM

munn

$110 \mathrm{mV}$

I $10 \mathrm{mV}$
Figure 7. Effect of the passive properties on the firing reliability of the different MNs. $A$, Change in the firing reliability in the different MNs in response to increased frequency of a sinusoidal current injection. $\boldsymbol{B}$, Graph showing the change in the firing of the different MNs as a function of the sinusoidal current frequency. $C$, Graph showing the change in the amplitude of the membrane potential depolarization as a function of the frequency of the injected current in the different pools of MNs.
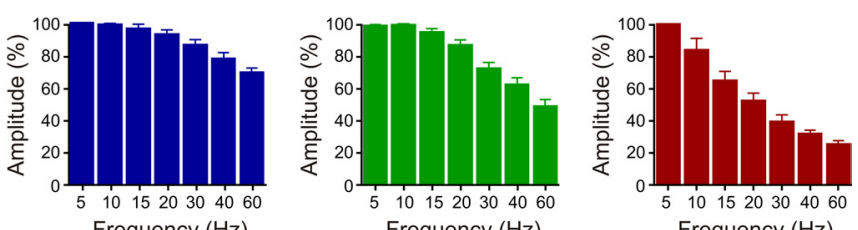

We previously showed that MNs are active during swimming according to a topographic pattern and that the threshold of recruitment is related to a combination of specific biophysical properties and the strength of the synaptic currents (Gabriel et al., 2011). The results of the present study extend and complement previous findings to show that MNs are organized into distinct modules, each with defined locations, properties, and recruitment patterns, and tuned to match the muscle properties and hence produce swimming at different speeds and modalities.

\section{Difference in organization and recruitment mechanisms} between larval and adult zebrafish

In zebrafish, the first motor activities with spontaneous coiling muscle contractions appear early at $17 \mathrm{~h}$ postfertilization (Kimmel et al., 1995), a time when only few MNs have extended their axons into the myotomes (Myers et al., 1986; Bernhardt et al., 1990; Kuwada and Bernhardt, 1990). At early larval stages [3 d postfertilization (dpf)], animals display swimming in erratic bursts (Buss and Drapeau, 2001) and at $4 \mathrm{dpf}$ a more directed beat-and-glide swimming pattern appears (Granato et al., 1996; Budick and O’Malley, 2000; Buss and Drapeau, 2001; Drapeau et al., 2002; Masino and Fetcho, 2005; 

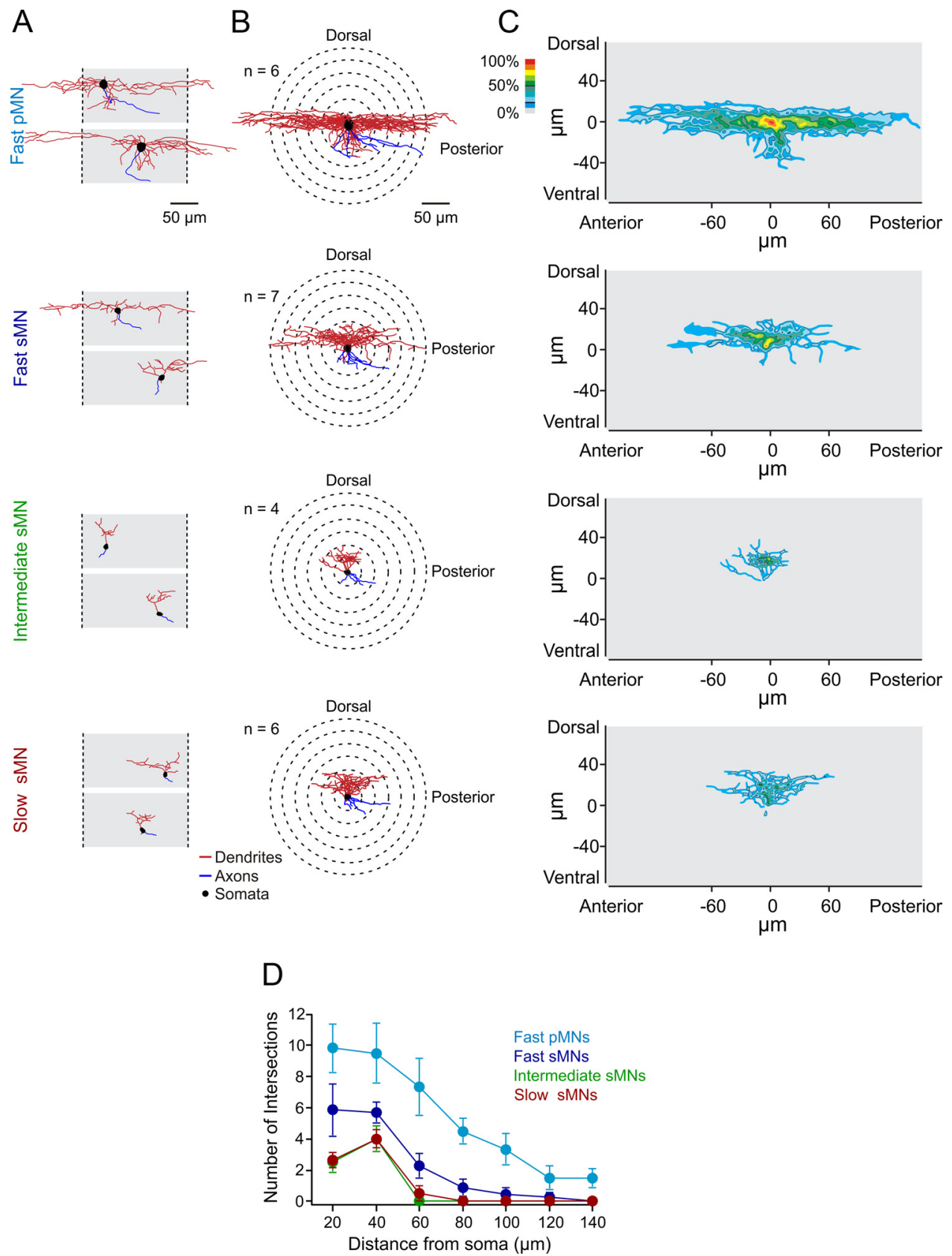

Figure 8. Morphological features of the different MN pools. $\boldsymbol{A}$, Representative examples of reconstructed MNs filled with neurobiotin. The position and orientation within the spinal cord segments (gray area) are represented with somas (black), axons (blue), and dendrites (red). B, Geometric maps of dendritic and axonal arbors of different MNs. The dendritic processes were aligned to the soma position with dotted concentric circles separated by $20 \mu \mathrm{m}$ distance for Sholl analysis. $\boldsymbol{C}$, Two-dimensional contour maps of dendritic arbors expressed as line density based on the digitized images after a Gaussian blur smoothing. Percentages refer to line density with $100 \%$ as maximum line density. D, Sholl analysis of the reconstructed MNs. The number of dendritic intersections plotted as a function of radial distance from the soma. The peak of the intersection curve lying $\sim 20-40 \mu \mathrm{m}$ from the soma. Vertical bars represent SEM.

Eklöf-Ljunggren et al., 2012). At this stage, only embryonic muscle fiber types are developed and a primary myotome is formed primarily by fast muscles fibers with only a superficial monolayer of slow muscle fibers (Devoto et al., 1996; Stellabotte and Devoto, 2007). As the zebrafish matures to the juvenile and the adult stages, the swimming activity increases and now the pattern changes to slow continuous swimming (Bone et al., 1978; van Raamsdonk et al., 1983; Müller and van Leeuwen, 2004; Kyriakatos et al., 2011; Ausborn et al., 2012). This change in the motor pattern is accompanied by the development of intermediate and adult slow red fibers (van Raamsdonk et al., 1982, 1983; Devoto et al., 1996). The trunk musculature in the adult zebrafish thus contains three types of muscle fibers with a specific arrangement into three discrete compartments: superficial slow fibers, an adjacent area of intermediate fibers, and more medially the larger part of the myotomes with fast fibers.

Our results show that the developmental changes in the muscle organization are associated with a refinement of MN organization. In larval zebrafish, only fast muscles are developed and they are innervated by early-developed fast pMNs and fast sMNs to produce motion geared for fast swimming and escape (Myers, 1985; Eisen et al., 1990; Pike and Eisen, 1990; McLean et al., 2007; Fetcho and McLean, 2010; Menelaou and McLean, 2012). At these early stages of development, the motor column extends only dorsoventrally in the spinal cord and is only $1-2$ cell bodies 
A

$$
\begin{gathered}
\text { Morphology } \\
(\mathrm{PC} 1+\mathrm{PC} 2=92.45 \%)
\end{gathered}
$$

B

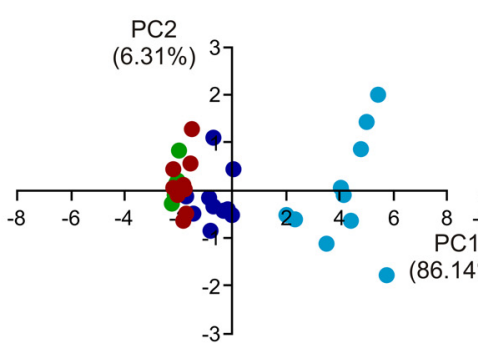

Physiology

$(\mathrm{PC} 1+\mathrm{PC} 2=74.80 \%)$

\section{$\mathrm{PC} 2$}

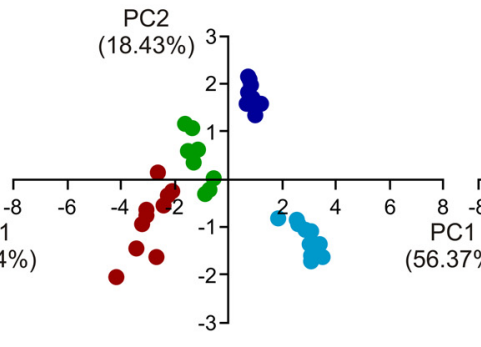

C
Morphology + Physiology

$(P C 1+P C 2=79.22 \%)$

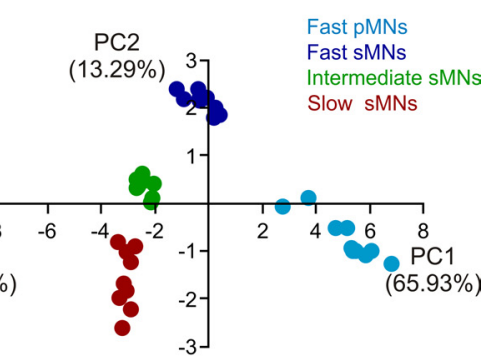

D Spinal cord Muscle types

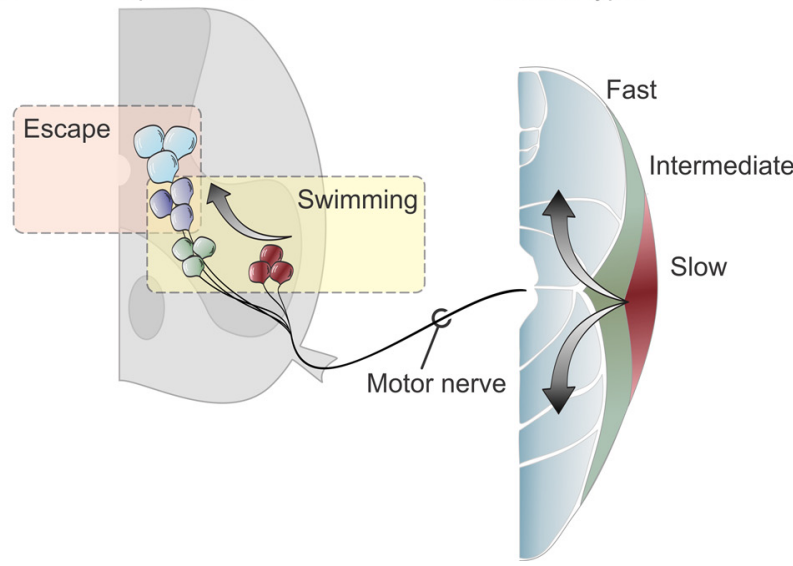

Figure 9. Morphological and physiological properties defining the different MN pools. $A$, PCA based on morphological features of the different MNs segregates sMNs from pMNs. $\boldsymbol{B}, \mathrm{PCA}$ analysis of physiological properties of the different MNs allows segregation of slow/intermediate from fast MNs. C, The combined PCA analysis of morphological and physiological properties allows distinction of slow, intermediate, and fast sMNs in addition to pMNs. D, Schematic summary of the organization and order of recruitment of the different MN pools in adult zebrafish.

thick. In addition, the properties of MNs lie in a continuum without any clear segregation into distinct pools as all these MNs innervate only fast muscles. The picture is clearly different in the adult zebrafish, where the intermediate and slow muscles are fully developed and the motor column extends laterally and more ventrally in the spinal cord (van Raamsdonk et al., 1983; Devoto et al., 1996). The present results extend our previous study (Gabriel et al., 2011) and show that the MN organization switches from the larval continuous pattern (Menelaou and McLean, 2012) into segregated pools displaying distinct anatomical and physiological properties with a clear somatotopic organization pattern related to the type of muscle they innervate. In addition, we show that the fast sMNs are shared between the escape and swimming circuits, that slow and intermediate sMNs are only used for swimming, and that fast pMNs are used only for escape.

\section{Modular principle of recruitment}

The size principle represents a conceptual framework that has attempted to explain the rules governing the recruitment of MNs (Henneman, 1957; Henneman and Mendell, 1981; Mendell, 2005). The prevailing view of this general principle is that motor units are recruited in an orderly manner from small highresistance to large low-resistance MNs (Henneman, 1957; Mendell, 2005). Most of the tests of this principle have been based on the size and conduction velocity of motor axons, which could not be directly related to the properties of MNs or the muscle fibers they innervate. Although the contribution of cellular properties were also considered to contribute to the recruitment order of MNs (Gustafsson and Pinter, 1984; Cope and Pinter, 1995), very few studies have addressed the mechanisms governing the re- cruitment order of MNs and their relationship with the properties of the target muscles during rhythmic activity of variable speed and strength.

Our results show that the order of recruitment of sMNs as a function of increased swimming speed in adult zebrafish is independent of their soma size or their input resistance. The recruitment order of sMNs is instead directly related to the innervated muscle type. Slow and intermediate MNs display almost identical morphological features and input resistance (Gabriel et al., 2011), but they are still not recruited simultaneously during swimming. Whether an $\mathrm{MN}$ will be recruited or not during swimming ultimately depends on the amplitude of the locomotor-driven oscillations (Gabriel et al., 2011). The oscillation amplitude is, however, not only a reflection of the synaptic drive, but it is also a computation of passive filtering and active conductances together with the synaptic drive. All these parameters are specifically tuned in the different MN pools to permit early recruitment of slow and intermediate MNs and delay that of fast MNs. Indeed, slow MNs display a low spike threshold, receive strong synaptic drive, and seem to display subthreshold conductances that contribute to their early recruitment. In contrast, fast MNs display a higher spike threshold and receive a weaker synaptic drive. This tunes their recruitment threshold toward higher swimming frequencies. Because of their depolarized spike threshold, fast MNs, when they are recruited, will display larger locomotor-related membrane potential oscillations compared with the slow and intermediate MNs. However, the large oscillation amplitude does not necessarily reflect the strength of synaptic transmission. Therefore, it is incorrect to infer the strength of the synaptic drive by simply analyzing the amplitude of these locomotor-related 
oscillations. Our results thus indicate that the recruitment order of sMNs during swimming is governed by an intricate sum of their intrinsic properties and synaptic drive that matches the properties of the target muscles, which are graded in a manner to confer with the muscle-type order of recruitment.

\section{Comparison of the organization of $\mathrm{MN}$ pools and muscle properties}

Traditionally the designation of MN pools does not take into account the contractile properties of the innervated muscle fibers and the developmental order of MNs (Romanes, 1964; Vanderhorst and Holstege, 1997). Thus, a motor pool usually encompasses a heterogeneous population of MNs (Romanes, 1964; Burke, 1981; Vanderhorst and Holstege, 1997; Dasen and Jessell, 2009). Our results suggest that the functional "unit motor pool" corresponds to a homogenous set of MNs innervating a homogenous set of muscle fibers. Each unit motor pool is endowed with specific position, morphological, and physiological properties that set their recruitment threshold to match the properties of the innervated muscle fibers. In mammals, most muscles contain heterogeneous motor unit populations with corresponding heterogeneous MN pools (Romanes, 1964; Burke, 1981; Vanderhorst and Holstege, 1997; Dasen and Jessell, 2009). The organization of these motor pools seems to reflect an anatomical and developmental proximity rather than a functional synergy. In this traditional view, motor pools may represent macropools related to the anatomical organization of the muscle they innervate. Our results suggest that each of these anatomically defined motor pools comprises subgroups of functionally different "unit motor pools" related to the contractile property of their motor units within a heterogeneous muscle. An implication of this arrangement is that the MNs belonging to functionally different unit motor pools innervating the same muscle would be connected to separate premotor interneurons and/or receive synaptic drive with variable strengths, depending on their recruitment order, with increased movement speed and force. This suggests that during evolution, there has been a fusion of muscles with different contractile properties to allow fine control of movements around multiple joints with a corresponding merging of unit motor pools into heterogeneous macropools. Therefore, the modular organization of $\mathrm{MN}$ pools revealed in our study and the rules governing their recruitment may also apply to other vertebrates where the heterogeneous muscles and their intermingled unit motor pools are inaccessible for such analysis.

\section{References}

Ausborn J, Mahmood R, El Manira A (2012) Decoding the rules of recruitment of excitatory interneurons in the adult zebrafish locomotor network. Proc Natl Acad Sci U S A 109:E3631-E3639. CrossRef Medline

Bender KJ, Rangel J, Feldman DE (2003) Development of columnar topography in the excitatory layer 4 to layer $2 / 3$ projection in rat barrel cortex. J Neurosci 23:8759-8770. Medline

Bernhardt RR, Chitnis AB, Lindamer L, Kuwada JY (1990) Identification of spinal neurons in the embryonic and larval zebrafish. J Comp Neurol 302:603-616. CrossRef Medline

Bone Q, Kiceniuk J, Jones DR (1978) On the role of the different fibre types in fish myotomes at intermediate swimming speeds. Fishery Bull 76:691-699.

Budick SA, O'Malley DM (2000) Locomotor repertoire of the larval zebrafish: swimming, turning and prey capture. J Exp Biol 203:2565-2579. Medline

Burke RE (1981) Motor units: anatomy, physiology, and functional organization. In: Handbook of physiology, section 1: the nervous system (Brooks VE, ed), pp 345-422. New York: Williams and Wilkins.

Büschges A, Scholz H, El Manira A (2011) New moves in motor control. Curr Biol 21:R513-R524. CrossRef Medline

Buss RR, Drapeau P (2001) Synaptic drive to motoneurons during fictive swimming in the developing zebrafish. J Neurophysiol 86:197-210. Medline

Cope TC, Pinter MJ (1995) The size principle: still working after all these years. News Physiol Sci 10:280-286.

Dasen JS, Jessell TM (2009) Hox networks and the origins of motor neuron diversity. Curr Top Dev Biol 88:169-200. CrossRef Medline

Devoto SH, Melançon E, Eisen JS, Westerfield M (1996) Identification of separate slow and fast muscle precursor cells in vivo, prior to somite formation. Development 122:3371-3380. Medline

Drapeau P, Saint-Amant L, Buss RR, Chong M, McDearmid JR, Brustein E (2002) Development of the locomotor network in zebrafish. Prog Neurobiol 68:85-111. CrossRef Medline

Eisen JS, Pike SH, Romancier B (1990) An identified motoneuron with variable fates in embryonic zebrafish. J Neurosci 10:34-43. Medline

Eklöf-Ljunggren E, Haupt S, Ausborn J, Dehnisch I, Uhlén P, Higashijima S, El Manira A (2012) Origin of excitation underlying locomotion in the spinal circuit of zebrafish. Proc Natl Acad Sci U S A 109:5511-5516. CrossRef Medline

Fetcho JR, McLean DL (2010) Some principles of organization of spinal neurons underlying locomotion in zebrafish and their implications. Ann N Y Acad Sci 1198:94-104. CrossRef Medline

Gabriel JP, Mahmood R, Walter AM, Kyriakatos A, Hauptmann G, Calabrese RL, El Manira A (2008) Locomotor pattern in the adult zebrafish spinal cord in vitro. J Neurophysiol 99:37-48. Medline

Gabriel JP, Mahmood R, Kyriakatos A, Söll I, Hauptmann G, Calabrese RL, El Manira A (2009) Serotonergic modulation of locomotion in zebrafish: endogenous release and synaptic mechanisms. J Neurosci 29:10387-10395. CrossRef Medline

Gabriel JP, Ausborn J, Ampatzis K, Mahmood R, Eklöf-Ljunggren E, El Manira A (2011) Principles governing recruitment of motoneurons during swimming in zebrafish. Nat Neurosci 14:93-99. CrossRef Medline Granato M, van Eeden FJ, Schach U, Trowe T, Brand M, Furutani-Seiki M, Haffter P, Hammerschmidt M, Heisenberg CP, Jiang YJ, Kane DA, Kelsh RN, Mullins MC, Odenthal J, Nüsslein-Volhard C (1996) Genes controlling and mediating locomotion behavior of the zebrafish embryo and larva. Development 123:399-413. Medline

Grillner S, Jessell TM (2009) Measured motion: searching for simplicity in spinal locomotor networks. Curr Opin Neurobiol 19:572-586. CrossRef Medline

Gustafsson B, Pinter MJ (1984) An investigation of threshold properties among cat spinal alpha-motoneurones. J Physiol 357:453-483. Medline

Henneman E (1957) Relation between size of neurons and their susceptibility to discharge. Science 126:1345-1347. CrossRef Medline

Henneman E, Mendell LM (1981) Functional organization of motoneuron pool and its inputs. In: Handbook of physiology, sect. 1, vol. 2 (Brooks VE, ed), pp 423-507. Bethesda, MD: American Physiological Society.

Kiehn O (2011) Development and functional organization of spinal locomotor circuits. Curr Opin Neurobiol 21:100-109. CrossRef Medline

Kimmel CB, Ballard WW, Kimmel SR, Ullmann B, Schilling TF (1995) Stages of embryonic development of the zebrafish. Dev Dyn 203:253-310. CrossRef Medline

Kuwada JY, Bernhardt RR (1990) Axonal outgrowth by identified neurons in the spinal cord of zebrafish embryos. Exp Neurol 109:29-34. CrossRef Medline

Kyriakatos A, Mahmood R, Ausborn J, Porres CP, Büschges A, El Manira A (2011) Initiation of locomotion in adult zebrafish. J Neurosci 31:84228431. CrossRef Medline

Lewis KE, Eisen JS (2003) From cells to circuits: development of the zebrafish spinal cord. Prog Neurobiol 69:419-449. CrossRef Medline

Masino MA, Fetcho JR (2005) Fictive swimming motor patterns in wild type and mutant larval zebrafish. J Neurophysiol 93:3177-3188. CrossRef Medline

McLean DL, Fan J, Higashijima S, Hale ME, Fetcho JR (2007) A topographic map of recruitment in spinal cord. Nature 446:71-75. CrossRef Medline

Meijering E, Jacob M, Sarria JC, Steiner P, Hirling H, Unser M (2004) Design and validation of a tool for neurite tracing and analysis in fluorescence microscopy images. Cytometry A 58:167-176. CrossRef Medline

Mendell LM (2005) The size principle: a rule describing the recruitment of motoneurons. J Neurophysiol 93:3024-3026. CrossRef Medline

Menelaou E, McLean DL (2012) A gradient in endogenous rhythmicity and oscillatory drive matches recruitment order in an axial motor pool. J Neurosci 32:10925-10939. CrossRef Medline 
Müller UK, van Leeuwen JL (2004) Swimming of larval zebrafish: ontogeny of body waves and implications for locomotory development. J Exp Biol 207:853-868. CrossRef Medline

Myers PZ (1985) Spinal motoneurons of the larval zebrafish. J Comp Neurol 236:555-561. CrossRef Medline

Myers PZ, Eisen JS, Westerfield M (1986) Development and axonal outgrowth of identified motoneurons in the zebrafish. J Neurosci 6:22782289. Medline

Pike SH, Eisen JS (1990) Identified primary motoneurons in embryonic zebrafish select appropriate pathways in the absence of other primary motoneurons. J Neurosci 10:44-49. Medline

Romanes GJ (1964) The motor pools of the spinal cord. Prog Brain Res 11:93-119. CrossRef Medline

Rossignol S, Dubuc R, Gossard JP (2006) Dynamic sensorimotor interactions in locomotion. Physiol Rev 86:89-154. CrossRef Medline

Sholl DA (1953) Dendritic organization in the neurons of the visual and motor cortices of the cat. J Anat 87:387-406. Medline

Sillar KT (2010) Development of spinal motor networks controlling axial movements. In: Oxford handbook of developmental behavioral neuroscience (Bumberh MS, Freeman JH, Robinson SR, eds), pp 240-253. Oxford: Oxford UP.
Stellabotte F, Devoto SH (2007) The teleost dermomyotome. Dev Dyn 236: 2432-2443. CrossRef Medline

van Raamsdonk W, van't Veer L, Veeken K, Heyting C, Pool CW (1982) Differentiation of muscle fiber types in the teleost Brachydanio rerio, the zebrafish. Posthatching development. Anat Embryol (Berl) 164: 51-62. CrossRef

van Raamsdonk W, Mos W, Smit-Onel MJ, van der Laarse WJ, Fehres R (1983) The development of the spinal motor column in relation to the myotomal muscle fibers in the zebrafish (Brachydanio rerio). I. Posthatching development. Anat Embryol (Berl) 167:125-139. CrossRef

Vanderhorst VG, Holstege G (1997) Organization of lumbosacral motoneuronal cell groups innervating hindlimb, pelvic floor, and axial muscles in the cat. J Comp Neurol 382:46-76. CrossRef Medline

Vinay L, Pearlstein E, Clarac F (2010) Development of spinal cord locomotor networks controlling limb movements. In: Oxford handbook of developmental behavioral neuroscience (Bumberh MS, Freeman JH, Robinson SR, eds), pp 210-239. Oxford: Oxford UP.

Waterman RE (1969) Development of the lateral musculature in the teleost Brachydanio rerio: a fine structural study. Am J Anat 125:457-493. CrossRef Medline 